\title{
Existence and uniqueness of solutions for delay boundary value problems with $p$-Laplacian on infinite intervals
}

\author{
Yuming Wei ${ }^{1 *}$ and Patricia JY Wong ${ }^{2}$
}

\section{"Correspondence:}

ymwei@gxnu.edu.cn

'School of Mathematical Science,

Guangxi Normal University, Guilin, 541004, China

Full list of author information is available at the end of the article

\begin{abstract}
This paper is concerned with the existence and uniqueness of solutions for boundary value problems with $p$-Laplacian delay differential equations on the half-line. The existence of solutions is derived from the Schauder fixed point theorem, whereas the uniqueness of solution is established by the Banach contraction principle. As an application, an example is given to demonstrate the main results.

MSC: 34K10; 34B18; 34B40
\end{abstract}

Keywords: delay differential equation; boundary value problem; infinite interval; Schauder fixed point theorem; Banach contraction principle

\section{Introduction}

Boundary value problems on infinite intervals have many applications in physical problems. Such problems arise, for example, in the study of linear elasticity, fluid flows and foundation engineering (see $[1,2]$ and the references cited therein). Boundary value problems on infinite intervals involving second-order delay differential equations are of specific interest in these applications. An interesting survey on infinite interval problems, including real world examples, history and various methods of proving solvability, can be found in the recent monograph by Agarwal et al. [3] and Agarwal and O'Regan [4]. Among the many articles dealing with boundary value problems of second-order delay differential equations, we refer the reader to [5] and the references cited therein.

Boundary value problems of second-order delay differential equations on infinite intervals are closely related to the problem of existence of global solutions with prescribed asymptotic behavior. Recently, there is a growing interest in the solutions of such boundary value problems; see, for example, [6-9]. For the basic theory of delay differential equations, the reader is referred to the books by Diekmann et al. [10] as well as by Hale and Verduyn Lunel [11]. For boundary value problems, we mention the monographs by Azbelev et al. [12] and Azbelev and Rakhmatullina [13].

To the best of our knowledge, few authors have considered the existence of solutions on infinite intervals for delay differential equations. As far as we know, only in $[6,8,9]$ the existence and uniqueness of solutions on infinite intervals for second-order delay boundary value problems are discussed. However, no work has been done on delay boundary value problems with $p$-Laplacian on infinite intervals. Motivated by the work mentioned above, this paper aims to fill in the gap, and we shall tackle the existence and uniqueness of

C 2013 Wei and Wong; licensee Springer. This is an Open Access article distributed under the terms of the Creative Commons Attribution License (http://creativecommons.org/licenses/by/2.0), which permits unrestricted use, distribution, and reproduction in any medium, provided the original work is properly cited. 
solutions to a boundary value problem of delay differential equation with $p$-Laplacian on infinite interval, which has been rarely discussed until now. The results we obtain improve and generalize the results mentioned in the references.

Throughout this paper, for any intervals $J$ and $X$ of $\mathbb{R}$, we denote by $C(J, X)$ the set of all continuous functions defined on $J$ with values in $X$. Let $r$ be a nonnegative real number. If $t$ is a point in the interval $[0, \infty)$ and $x$ is a continuous real-valued function defined at least on $[t-r, t]$, the notation $x_{t}$ will be used for the function in $C([-r, 0], \mathbb{R})$ defined by

$$
x_{t}(\sigma)=x(t+\sigma) \quad \text { for }-r \leq \sigma \leq 0 .
$$

We notice that the set $C([-r, 0], \mathbb{R})$ is a Banach space equipped with the usual sup-norm $\|\cdot\|$ given by

$$
\|\psi\|=\max _{-r \leq \sigma \leq 0}|\psi(\sigma)| \quad \text { for } \psi \in C([-r, 0], \mathbb{R}) .
$$

In this paper we consider the delay differential equation with $p$-Laplacian

$$
\left(\phi_{p}\left(x^{\prime}(t)\right)\right)^{\prime}+f\left(t, x_{t}, x^{\prime}(t)\right)=0, \quad t \geq 0,
$$

subject to

$$
x(t)=\xi(t), \quad-r \leq t \leq 0
$$

and

$$
\lim _{t \rightarrow \infty} x^{\prime}(t)=0
$$

where $p>1, \phi_{p}(s)=|s|^{p-2} s, \xi \in C([-r, 0], \mathbb{R})$ with $\xi(0)=0$, and $f$ is a real-valued function defined on the set $[0, \infty) \times C([-r, 0], \mathbb{R}) \times \mathbb{R}$, which satisfies the following continuity condition: $f\left(t, x_{t}, x^{\prime}(t)\right)$ is continuous with respect to $t$ in $[0, \infty)$ for each given function $x \in C([-r, \infty), \mathbb{R})$ which is continuously differentiable on the interval $[0, \infty)$.

We are interested in global solutions of the $p$-Laplacian delay boundary value problem (1.1)-(1.3). By a solution on $[0, \infty)$ of (1.1)-(1.3), we mean a function $x \in C([-r, \infty), \mathbb{R})$ which is continuously differentiable on the interval $[0, \infty)$ such that $(1.1)$ is satisfied for all $t \geq 0$ and the conditions (1.2) and (1.3) are also fulfilled.

The main results of this paper are stated in Section 2. In Theorem 2.1, sufficient conditions are established in order that (1.1)-(1.3) has at least one solution on $[0, \infty)$, whereas Theorem 2.2 provides sufficient conditions for (1.1)-(1.3) to have a unique solution on $[0, \infty)$. The proof of Theorem 2.1 and Theorem 2.2 is presented in Section 3, where we employ the Schauder fixed point theorem and the well known Banach contraction principle. In Section 4, we include an example to illustrate our main results.

\section{Main results}

A useful integral representation of the boundary value problem (1.1)-(1.3) is given by the following lemma. We note that $\phi_{p}(s)=|s|^{p-2} s$ has the inverse $\phi_{p}^{-1}(s)=\phi_{q}(s)$, where $\frac{1}{p}+\frac{1}{q}=1$. 
Lemma 2.1 Let $x$ be a function in $C([-r, \infty), \mathbb{R})$ that is continuously differentiable on $[0, \infty)$. Then $x$ is a solution on $[0, \infty)$ of the boundary value problem (1.1)-(1.3) if and only if

$$
x(t)= \begin{cases}\xi(t) & \text { for }-r \leq t \leq 0, \\ \int_{0}^{t} \phi_{q}\left(\int_{\theta}^{\infty} f\left(s, x_{s}, x^{\prime}(s)\right) d s\right) d \theta & \text { for } t \geq 0 .\end{cases}
$$

Proof Let $x$ be a function in $C([-r, \infty), \mathbb{R})$ that is continuously differentiable on $[0, \infty)$. Assume that $x$ is given by (2.1). Then (1.2) is fulfilled. Moreover, we immediately obtain

$$
x^{\prime}(t)=\phi_{q}\left(\int_{t}^{\infty} f\left(s, x_{s}, x^{\prime}(s)\right) d s\right), \quad t \geq 0,
$$

which implies that $\lim _{t \rightarrow \infty} x^{\prime}(t)=0$, i.e., (1.3) holds. Furthermore, from (2.2) we get

$$
\phi_{p}\left(x^{\prime}(t)\right)=\int_{t}^{\infty} f\left(s, x_{s}, x^{\prime}(s)\right) d s, \quad t \geq 0
$$

and we have

$$
\left(\phi_{p}\left(x^{\prime}(t)\right)\right)^{\prime}=-f\left(t, x_{t}, x^{\prime}(t)\right), \quad t \geq 0,
$$

which means that $x$ satisfies (1.1). Thus, $x$ is a solution on $[0, \infty)$ of the boundary value problem (1.1)-(1.3).

Conversely, suppose that $x$ is a solution on $[0, \infty)$ of the boundary value problem (1.1)(1.3). In view of (1.2), we have $x(t)=\xi(t)$ for $-r \leq t \leq 0$. Furthermore, from (1.1) it follows that $x$ satisfies (2.4). Taking into account the fact that $\lim _{t \rightarrow \infty} x^{\prime}(t)=0$ and $\phi_{p}(0)=0$, consequently we have

$$
\phi_{p}\left(x^{\prime}(t)\right)=\int_{t}^{\infty} f\left(s, x_{s}, x^{\prime}(s)\right) d s, \quad t \geq 0 .
$$

It follows that

$$
x^{\prime}(t)=\phi_{q}\left(\int_{t}^{\infty} f\left(s, x_{s}, x^{\prime}(s)\right) d s\right), \quad t \geq 0 .
$$

By integrating (2.6) and taking into account the fact that $x(0)=\xi(0)=0$, we obtain for every $t \geq 0$,

$$
x(t)=\int_{0}^{t} \phi_{q}\left(\int_{\theta}^{\infty} f\left(s, x_{s}, x^{\prime}(s)\right) d s\right) d \theta .
$$

We have thus proved that $x$ has the expression (2.1). The proof of the lemma is complete.

The first main result of this paper is the following theorem which provides sufficient conditions for (1.1)-(1.3) to have at least one solution on $[0, \infty)$. 
Theorem 2.1 Suppose that

$$
|f(t, \psi, z)| \leq F(t,|\psi|,|z|) \quad \text { for }(t, \psi, z) \in[0, \infty) \times C([-r, 0], \mathbb{R}) \times \mathbb{R}
$$

where $F$ is a nonnegative real-valued function defined on $[0, \infty) \times C([-r, 0],[0, \infty)) \times$ $[0, \infty)$ which satisfies the continuity condition:

(C) $F\left(t,\left|x_{t}\right|,\left|x^{\prime}(t)\right|\right)$ is continuous with respect to $t$ in $[0, \infty)$ for each given function $x$ in $C([-r, \infty), \mathbb{R})$ which is continuously differentiable on $[0, \infty)$.

We also assume that

(A) for each $t>0$, the function $F(t, \cdot, \cdot)$ is increasing on $C([-r, 0],[0, \infty)) \times[0, \infty)$ in the sense that $F(t, \psi, z) \leq F(t, \omega, v)$ for any $\psi, \omega \in C([-r, 0],[0, \infty))$ with $\psi \leq \omega$ (i.e., $\psi(\tau) \leq \omega(\tau)$ for $-r \leq \tau \leq 0)$ and any $z, v \in[0, \infty)$ with $z \leq v$. Moreover, there exists a real number $c>0$ so that

$$
\int_{0}^{\infty} F\left(t, \eta_{t}, c\right) d t \leq \phi_{p}(c)=c^{p-1}
$$

where the function $\eta \in C([-r, \infty),[0, \infty))$ depends on $\xi, c$ and is defined by

$$
\eta(t)= \begin{cases}|\xi(t)| & \text { for }-r \leq t \leq 0 \\ c t & \text { for } t \geq 0\end{cases}
$$

Then the boundary value problem (1.1)-(1.3) has at least one solution $x$ on $[0, \infty)$ such that

$$
-c t \leq x(t) \leq c t \quad \text { for } t \geq 0
$$

and

$$
-c \leq x^{\prime}(t) \leq c \text { for } t \geq 0 .
$$

The second main result is the following theorem that establishes conditions under which the boundary value problem (1.1)-(1.3) has a unique solution on $[0, \infty)$.

Theorem 2.2 Let all the conditions of Theorem 2.1 be satisfied, i.e., (2.7), (C) and (A) hold. Moreover, suppose that

$$
\begin{aligned}
& \left|\phi_{q}\left(\int_{t}^{\infty} f(s, \psi, z) d s\right)-\phi_{q}\left(\int_{t}^{\infty} f(s, \omega, v) d s\right)\right| \\
& \quad \leq K(t) \max \{\|\psi-\omega\|,|z-v|\} \\
& \quad \text { for }(t, \psi, z),(t, \omega, v) \in[0, \infty) \times C([-r, 0], \mathbb{R}) \times \mathbb{R},
\end{aligned}
$$

where $K$ is a nonnegative continuous real-valued function on the interval $[0, \infty)$ satisfying

$$
K(t) \max \{t, 1\}<1 .
$$

Then the boundary value problem (1.1)-(1.3) has a unique solution $x$ on $[0, \infty)$ satisfying (2.10) and (2.11). 


\section{Proof of main results}

To prove Theorem 2.1, we shall use the fixed point technique by applying the Schauder fixed point theorem [14], whereas Theorem 2.2 is established by the Banach contraction principle [15]. We state our main tools below.

Schauder fixed point theorem [14] Let E be a Banach space and $\Omega$ be any nonempty convex and closed subset of E. If $M$ is a continuous mapping of $\Omega$ into itself and $M \Omega$ is relatively compact, then the mapping $M$ has at least one fixed point, i.e., there exists an $x \in \Omega$ such that $x=M x$.

Let $B C([0, \infty), \mathbb{R})$ be the Banach space of all bounded continuous real-valued functions on $[0, \infty)$, endowed with the sup-norm $\|\cdot\|$ given by

$$
\|\psi\|=\sup _{t \geq 0}|\psi(t)| \quad \text { for } \psi \in B C([0, \infty), \mathbb{R}) .
$$

We need the following compactness criterion for a subset of $B C([0, \infty), \mathbb{R})$, which is a consequence of the well-known Arzela-Ascoli theorem. This compactness criterion is an adaptation of a lemma due to Avramescu [16]. In order to formulate this criterion, we note that a set $U$ of real-valued functions defined on $[0, \infty)$ is said to be equiconvergent at $\infty$ if all the functions in $U$ are convergent in $\mathbb{R}$ at the point $\infty$ and, in addition, for each $\epsilon>0$, there exists $T \equiv T(\epsilon)>0$ such that, for any function $\psi \in U$, we have $\left|\psi(t)-\lim _{s \rightarrow \infty} \psi(s)\right|<$ $\epsilon$ for $t \geq T$.

Compactness criterion [16] Let $U$ be an equicontinuous and uniformly bounded subset of the Banach space $B C([0, \infty), \mathbb{R})$. If $U$ is equiconvergent at $\infty$, it is also relatively compact.

Banach contraction principle [15] Let $E$ be a Banach space and $\Omega$ be any nonempty closed subset of $E$. If $M$ is a contraction of $\Omega$ into itself, then the mapping $M$ has a unique fixed point, i.e., there exists a unique $x \in \Omega$ such that $x=M x$.

Throughout this section, let $E$ denote the set of all functions $x$ in $C([-r, \infty), \mathbb{R})$, which are continuously differentiable on the interval $[0, \infty)$ and have bounded continuous derivatives on $[0, \infty)$. The set $E$ is a Banach space endowed with the norm $\|\cdot\|_{E}$ given by

$$
\|x\|_{E}=\max \left\{\max _{-r \leq t \leq 0}|x(t)|, \sup _{t \geq 0}\left|x^{\prime}(t)\right|\right\} \quad \text { for } x \in E .
$$

We shall first establish a lemma which will be needed to prove the main results.

Lemma 3.1 Suppose that (2.7) holds, where $F$ is a nonnegative real-valued function defined on $[0, \infty) \times C([-r, 0],[0, \infty)) \times[0, \infty)$, which satisfies the continuity condition $(C)$. Assume that (A) is satisfied. Let $\Omega$ be the subset of the Banach space E defined by

$$
\Omega=\left\{x \in E: x(t)=\xi(t) \text { for }-r \leq t \leq 0,-c \leq x^{\prime}(t) \leq c \text { for } t \geq 0\right\} .
$$

For $x \in \Omega$, define a mapping $M$ on $\Omega$ by

$$
(M x)(t)= \begin{cases}\xi(t) & \text { for }-r \leq t \leq 0, \\ \int_{0}^{t} \phi_{q}\left(\int_{\theta}^{\infty} f\left(s, x_{s}, x^{\prime}(s)\right) d s\right) d \theta & \text { for } t \geq 0 .\end{cases}
$$


Then $M$ maps $\Omega$ into E. Moreover, $M \Omega$ is relatively compact and the mapping $M: \Omega \rightarrow E$ is continuous.

Proof Since the condition (A) is satisfied, from (2.8) we have

$$
\int_{0}^{\infty} F\left(t, \eta_{t}, c\right) d t \leq \phi_{p}(c)=c^{p-1}<\infty .
$$

First, we shall show that $M$ is a mapping from $\Omega$ into $E$, i.e., $M \Omega \subseteq E$. Let $x$ be an arbitrary function in $\Omega$. By the definition of $\Omega$, the function $x$ satisfies (1.2) and (2.11). Since $\xi(0)=0$, it follows from (1.2) that $x(0)=0$. By taking into account this fact and using (2.11), we can easily obtain

$$
|x(t)| \leq c t \quad \text { for } t \geq 0 \text {. }
$$

By virtue of (1.2), (3.3) and (2.9), it follows that $|x(t)| \leq \eta(t)$ for all $t \geq-r$, which ensures that

$$
\left|x_{t}\right| \leq \eta_{t} \quad \text { for } t \geq 0 .
$$

In view of (3.4), (2.11) and the condition (A), we get

$$
F\left(t,\left|x_{t}\right|,\left|x^{\prime}(t)\right|\right) \leq F\left(t, \eta_{t}, c\right) \quad \text { for } t \geq 0 .
$$

On the other hand, (2.7) guarantees that

$$
\left|f\left(t, x_{t}, x^{\prime}(t)\right)\right| \leq F\left(t,\left|x_{t}\right|,\left|x^{\prime}(t)\right|\right) \quad \text { for } t \geq 0 .
$$

Thus, we have

$$
\left|f\left(t, x_{t}, x^{\prime}(t)\right)\right| \leq F\left(t, \eta_{t}, c\right) \quad \text { for } t \geq 0 .
$$

From (3.2) and (3.5) it follows that

$$
\int_{0}^{\infty}\left|f\left(t, x_{t}, x^{\prime}(t)\right)\right| d t<\infty
$$

and consequently,

$$
\int_{0}^{\infty} f\left(t, x_{t}, x^{\prime}(t)\right) d t \quad \text { exists in } \mathbb{R} \text {. }
$$

Furthermore, we can conclude that

$$
\int_{0}^{t} \phi_{q}\left(\int_{\theta}^{\infty} f\left(s, x_{s}, x^{\prime}(s)\right) d s\right) d \theta \text { exists in } \mathbb{R} .
$$

Since (3.8) holds for any function $x \in \Omega$, we immediately see that the formula (3.1) makes sense for any $x \in \Omega$, and this formula defines a mapping $M$ from $\Omega$ into $C([-r, \infty), \mathbb{R})$. 
Next, using (3.5) and (2.8), from (3.1) we obtain for $t \geq 0$,

$$
\begin{aligned}
\left|(M x)^{\prime}(t)\right| & =\left|\phi_{q}\left(\int_{t}^{\infty} f\left(s, x_{s}, x^{\prime}(s)\right) d s\right)\right| \\
& \leq \phi_{q}\left(\int_{t}^{\infty}\left|f\left(s, x_{s}, x^{\prime}(s)\right)\right| d s\right) \\
& \leq \phi_{q}\left(\int_{0}^{\infty}\left|f\left(s, x_{s}, x^{\prime}(s)\right)\right| d s\right) \\
& \leq \phi_{q}\left(\int_{0}^{\infty} F\left(s, \eta_{s}, c\right) d s\right) \\
& \leq \phi_{q}\left(\phi_{p}(c)\right)=c .
\end{aligned}
$$

Inequality (3.9) means that $(M x)^{\prime}$ is bounded on the interval $[0, \infty)$ and so $M x$ belongs to $E$. We have thus proved that $M \Omega \subseteq E$.

Now, we shall prove that $M \Omega$ is relatively compact. We observe that, for any function $x \in \Omega$, we have $(M x)(t)=\xi(t)$ for $-r \leq t \leq 0$. By taking into account this fact as well as the definition of the norm $\|\cdot\|_{E}$, we can easily conclude that it suffices to show that the set

$$
U=\left\{\left.(M x)^{\prime}(t)\right|_{t \in[0, \infty)}: x \in \Omega\right\}
$$

is relatively compact in the Banach space $B C([0, \infty), \mathbb{R})$. Using $(3.5)$, for any $x \in \Omega$ and any $t_{1}, t_{2}$ with $0 \leq t_{1} \leq t_{2}$, we obtain

$$
\begin{aligned}
\left|\phi_{p}\left((M x)^{\prime}\left(t_{1}\right)\right)-\phi_{p}\left((M x)^{\prime}\left(t_{2}\right)\right)\right| & =\left|\int_{t_{1}}^{\infty} f\left(s, x_{s}, x^{\prime}(s)\right) d s-\int_{t_{2}}^{\infty} f\left(s, x_{s}, x^{\prime}(s)\right) d s\right| \\
& =\left|\int_{t_{1}}^{t_{2}} f\left(s, x_{s}, x^{\prime}(s)\right) d s\right| \\
& \leq \int_{t_{1}}^{t_{2}}\left|f\left(s, x_{s}, x^{\prime}(s)\right)\right| d s \\
& \leq \int_{t_{1}}^{t_{2}} F\left(s, \eta_{s}, c\right) d s .
\end{aligned}
$$

In view of (3.2), this means that $(M x)^{\prime}\left(t_{1}\right) \rightarrow(M x)^{\prime}\left(t_{2}\right)$ as $t_{1} \rightarrow t_{2}$, and we can easily verify that $U$ is equicontinuous. Moreover, each function $x \in \Omega$ satisfies (3.9), where $c$ is independent of $x$. This guarantees that $U$ is uniformly bounded. Furthermore, for any $x \in \Omega$, we have

$$
\left|(M x)^{\prime}(t)\right|=\left|\phi_{q}\left(\int_{t}^{\infty} f\left(s, x_{s}, x^{\prime}(s)\right) d s\right)\right| \leq \phi_{q}\left(\int_{t}^{\infty}\left|f\left(s, x_{s}, x^{\prime}(s)\right)\right| d s\right) \quad \text { for } t \geq 0
$$

and hence, noting (3.5), it follows that

$$
\left|(M x)^{\prime}(t)\right| \leq \phi_{q}\left(\int_{t}^{\infty} F\left(s, \eta_{s}, c\right) d s\right) \text { for } t \geq 0
$$

Now (3.10) together with (3.2) implies that

$$
\lim _{t \rightarrow \infty}(M x)^{\prime}(t)=0
$$


By using (3.2) and (3.10) again, we immediately see that $U$ is equiconvergent at $\infty$. It now follows from the given compactness criterion that the set $U$ is relatively compact in $B C([0, \infty), \mathbb{R})$.

Finally, we shall prove that the mapping $M: \Omega \rightarrow E$ is continuous. Let $x,\left\{x^{n}\right\}_{n \geq 1} \in \Omega$ with $\left\|x^{n}-x\right\|_{E} \rightarrow 0$ as $n \rightarrow \infty$. It is not difficult to verify that $\lim _{n \rightarrow \infty} x^{n}(t)=x(t)$ uniformly for $t \in[-r, \infty)$ and $\lim _{n \rightarrow \infty}\left(x^{n}\right)^{\prime}(t)=x^{\prime}(t)$ uniformly for $t \in[0, \infty)$. On the other hand, using (3.5) we have

$$
\left|f\left(t, x_{t}^{n},\left(x^{n}\right)^{\prime}(t)\right)\right| \leq F\left(t, \eta_{t}, c\right) \text { for } t \geq 0 \text { and } n \geq 1
$$

Thus, by taking into account the fact that

$$
\int_{0}^{t} \phi_{q}\left(\int_{\theta}^{\infty} F\left(s, \eta_{s}, c\right) d s\right) d \theta<\infty
$$

we can apply the Lebesgue dominated convergence theorem to obtain, for every $t \geq 0$,

$$
\lim _{n \rightarrow \infty} \int_{0}^{t} \phi_{q}\left(\int_{\theta}^{\infty} f\left(s, x_{s}^{n},\left(x^{n}\right)^{\prime}(s)\right) d s\right) d \theta=\int_{0}^{t} \phi_{q}\left(\int_{\theta}^{\infty} f\left(s, x_{s}, x^{\prime}(s)\right) d s\right) d \theta .
$$

This, together with the fact that

$$
\left(M x^{n}\right)(t)=(M x)(t)=\xi(t) \text { for }-r \leq t \leq 0 \text { and } n \geq 1,
$$

guarantees the pointwise convergence

$$
\lim _{n \rightarrow \infty}\left(M x^{n}\right)(t)=(M x)(t) \quad \text { for } t \geq-r .
$$

It remains to show that this convergence is also convergence in the sense of $\|\cdot\|_{E}$, i.e.,

$$
\lim _{n \rightarrow \infty}\left\|M x^{n}-M x\right\|_{E}=0 .
$$

For this purpose, we consider an arbitrary subsequence $\left\{M x^{k}\right\}$ of $\left\{M x^{n}\right\}$. Since $M \Omega$ is relatively compact, there exists a subsequence $\left\{M x^{j}\right\}$ of the sequence $\left\{M x^{k}\right\}$ and a function $u$ in $E$ so that $\lim _{j \rightarrow \infty}\left\|M x^{j}-u\right\|_{E}=0$. As the convergence in the sense of $\|\cdot\|_{E}$ implies the pointwise convergence to the same limit function, we must have $u=M x$, therefore (3.11) holds. Consequently, $M$ is continuous. The proof is complete.

Proof of Theorem 2.1 We shall apply the Schauder fixed point theorem. Let $\Omega$ be the subset of the Banach space $E$ defined as in Lemma 3.1. Clearly, $\Omega$ is a nonempty convex and closed subset of $E$. By Lemma 3.1, the mapping $M: \Omega \rightarrow E$ is continuous and $M \Omega$ is relatively compact. We shall show that $M$ maps $\Omega$ into itself, i.e., $M \Omega \subseteq \Omega$. Let us consider an arbitrary function $x \in \Omega$. Following the argument in the proof of Lemma 3.1, we see that $x$ satisfies (3.10), which together with (2.8) provides

$$
\left|(M x)^{\prime}(t)\right| \leq \phi_{q}\left(\int_{t}^{\infty} F\left(s, \eta_{s}, c\right) d s\right) \leq \phi_{q}\left(\phi_{p}(c)\right)=c \quad \text { for } t \geq 0 .
$$


Now, (3.12) and the fact that $(M x)(t)=\xi(t)$ for $-r \leq t \leq 0$ imply that $M x \in \Omega$. We have thus proved that $M \Omega \subseteq \Omega$.

By the Schauder fixed point theorem, there exists an $x \in \Omega$ such that $x=M x$. Hence, $x$ has the expression (3.1), which coincides with (2.1). It follows from Lemma 2.1 that $x$ is a solution on $[0, \infty)$ of the boundary value problem (1.1)-(1.3). Also, since $x \in \Omega$, clearly $x$ satisfies (2.11). Moreover, since $x(0)=\xi(0)=0$, it follows from (2.11) that $x$ also fulfills (2.10). This completes the proof of Theorem 2.1.

Proof of Theorem 2.2 We shall employ the Banach contraction principle. Let $\Omega$ be the subset of the Banach space $E$ defined in Lemma 3.1. Clearly, $\Omega$ is a nonempty closed subset of $E$. Following the argument in the proof of Theorem 2.1, we have $M: \Omega \rightarrow \Omega$.

Now, we shall prove that the mapping $M$ is a contraction. For this purpose, let us consider two arbitrary functions $x$ and $\tilde{x}$ in $\Omega$. From (3.1), we have $(M x)(t)=(M \tilde{x})(t)=\xi(t)$ for $-r \leq t \leq 0$, and consequently,

$$
\max _{-r \leq t \leq 0}|(M x)(t)-(M \tilde{x})(t)|=0 .
$$

Furthermore, by using (2.12), from (3.1) we obtain for $t \geq 0$

$$
\begin{aligned}
\left|(M x)^{\prime}(t)-(M \tilde{x})^{\prime}(t)\right| & =\left|\phi_{q}\left(\int_{t}^{\infty} f\left(s, x_{s}, x^{\prime}(s)\right) d s\right)-\phi_{q}\left(\int_{t}^{\infty} f\left(s, \tilde{x}_{s}, \tilde{x}^{\prime}(s)\right) d s\right)\right| \\
& \leq K(t) \max \left\{\left\|x_{s}-\tilde{x}_{s}\right\|,\left|x^{\prime}(s)-\tilde{x}^{\prime}(s)\right|\right\} .
\end{aligned}
$$

This gives

$$
\sup _{t \geq 0}\left|(M x)^{\prime}(t)-(M \tilde{x})^{\prime}(t)\right| \leq \sup _{t \geq 0} K(t) \max \left\{\left\|x_{t}-\tilde{x}_{t}\right\|,\left|x^{\prime}(t)-\tilde{x}^{\prime}(t)\right|\right\} .
$$

By the definition of the norm $\|\cdot\|_{E}$, the last inequality and (3.13) imply

$$
\|M x-M \tilde{x}\|_{E} \leq \sup _{t \geq 0} K(t) \max \left\{\left\|x_{t}-\tilde{x}_{t}\right\|,\left|x^{\prime}(t)-\tilde{x}^{\prime}(t)\right|\right\} .
$$

Next, from the definition of $\Omega$, we have $x(t)=\tilde{x}(t)=\xi(t)$ for $-r \leq t \leq 0$, and so

$$
|x(t)-\tilde{x}(t)|=0 \quad \text { for }-r \leq t \leq 0 .
$$

Moreover, in view of the fact that $x(0)=\tilde{x}(0)=\xi(0)=0$, we get, for $t \geq 0$,

$$
|x(t)-\tilde{x}(t)|=\left|\int_{0}^{t}\left(x^{\prime}(s)-\tilde{x}^{\prime}(s)\right) d s\right| \leq \int_{0}^{t}\left|x^{\prime}(s)-\tilde{x}^{\prime}(s)\right| d s .
$$

But, by the definition of the norm $\|\cdot\|_{E}$, we have

$$
\left|x^{\prime}(t)-\tilde{x}^{\prime}(t)\right| \leq\|x-\tilde{x}\|_{E} \quad \text { for } t \geq 0 .
$$

Thus, using (3.17) in (3.16) yields

$$
|x(t)-\tilde{x}(t)| \leq t\|x-\tilde{x}\|_{E} \quad \text { for } t \geq 0 .
$$


Combining (3.15) and (3.18), we get

$$
|x(t)-\tilde{x}(t)| \leq \mu(t)\|x-\tilde{x}\|_{E} \quad \text { for } t \geq-r,
$$

where the function $\mu$ is defined by

$$
\mu(t)= \begin{cases}0 & \text { for }-r \leq t \leq 0 \\ t & \text { for } t \geq 0\end{cases}
$$

We can rewrite (3.19) as

$$
|x(t+\tau)-\tilde{x}(t+\tau)| \leq \mu(t+\tau)\|x-\tilde{x}\|_{E} \quad \text { for } t \geq 0 \text { and }-r \leq \tau \leq 0 \text {, }
$$

i.e.,

$$
\left|x_{t}(\tau)-\tilde{x}_{t}(\tau)\right| \leq \mu(t+\tau)\|x-\tilde{x}\|_{E} \quad \text { for } t \geq 0 \text { and }-r \leq \tau \leq 0 \text {. }
$$

It follows that

$$
\max _{-r \leq \tau \leq 0}\left|x_{t}(\tau)-\tilde{x}_{t}(\tau)\right| \leq\left[\max _{-r \leq \tau \leq 0} \mu(t+\tau)\right]\|x-\tilde{x}\|_{E} \quad \text { for } t \geq 0
$$

But, since $\mu$ is nondecreasing on $[-r, \infty)$, we have

$$
\max _{-r \leq \tau \leq 0} \mu(t+\tau)=\mu(t)=t \quad \text { for } t \geq 0 .
$$

So, from (3.20) we have

$$
\left\|x_{t}-\tilde{x}_{t}\right\| \leq t\|x-\tilde{x}\|_{E} \quad \text { for } t \geq 0 .
$$

Now, using (3.17) and (3.21) in (3.14), we get

$$
\begin{aligned}
\|M x-M \tilde{x}\|_{E} & \leq \sup _{t \geq 0} K(t) \max \left\{t\|x-\tilde{x}\|_{E},\|x-\tilde{x}\|_{E}\right\} \\
& =\sup _{t \geq 0} K(t) \max \{t, 1\}\|x-\tilde{x}\|_{E} \\
& <\|x-\tilde{x}\|_{E},
\end{aligned}
$$

where the last inequality is due to (2.13). Hence, we have shown that the mapping $M: \Omega \rightarrow$ $\Omega$ is a contraction.

Finally, by the Banach contraction principle, the mapping $M: \Omega \rightarrow \Omega$ has a unique fixed point $x \in \Omega$ having the expression (3.1), which coincides with (2.1). It follows from Lemma 2.1 that $x$ is the unique solution on $[0, \infty)$ of the boundary value problem (1.1)(1.3). Furthermore, as in the proof of Theorem 2.1, we conclude that this unique solution $x$ of the boundary value problem (1.1)-(1.3) satisfies (2.10) and (2.11). The proof of Theorem 2.2 is now complete. 


\section{Application}

Let us consider the delay boundary value problem with the $p$-Laplacian operator

$$
\left\{\begin{array}{l}
\left(\phi_{p}\left(x^{\prime}(t)\right)\right)^{\prime}+h\left(t, x(t-T(t)), x^{\prime}(t)\right)=0, \quad 0 \leq t<\infty \\
x(t)=\xi(t), \quad-r \leq t \leq 0 \\
\lim _{t \rightarrow \infty} x^{\prime}(t)=0
\end{array}\right.
$$

where $p=2,\left(\phi_{p}\left(x^{\prime}(t)\right)\right)^{\prime}=x^{\prime \prime}(t)$, and $h$ is a continuous real-valued function on $[0, \infty) \times$ $\mathbb{R}^{2}$, and $T(t)$ is a nonnegative continuous real-value function on the interval $[0, \infty)$ with $\sup _{t \geq 0} T(t)=r$.

If the boundary value problem (1.1)-(1.3) is to be equivalent to the boundary value problem (4.1), we must define $f(t, \psi, z)=h(t, \psi(-T(t)), z)$ for $(t, \psi, z) \in[0, \infty) \times C([-r, 0]$, $\mathbb{R}) \times \mathbb{R}$. Hence, by applying Theorem 2.1 to the boundary value problem (4.1), we can be led to the following result.

Corollary 4.1 Assume that

$$
|h(t, y, z)| \leq H(t,|y|,|z|) \quad \text { for }(t, y, z) \in[0, \infty) \times \mathbb{R} \times \mathbb{R}
$$

where $H$ is a nonnegative continuous real-valued function on $[0, \infty) \times[0, \infty) \times[0, \infty)$. Suppose that for each $t \geq 0$, the function $H(t, \cdot, \cdot)$ is increasing on $[0, \infty) \times[0, \infty)$ in the sense that $H(t, y, z) \leq H(t, w, v)$ for any $(y, z),(w, v) \in[0, \infty) \times[0, \infty)$ with $y \leq w$ and $z \leq v$.

Moreover, let there exist a real number $c>0$ so that

$$
\int_{0}^{\infty} H(t, \rho(t), c) d t \leq c
$$

where the function $\rho(t)$ in $C([0, \infty),[0, \infty))$ depends on $\xi$, c and is defined by

$$
\rho(t)= \begin{cases}|\xi(t-T(t))| & \text { for } 0 \leq t \leq T(t) \\ c \cdot(t-T(t)) & \text { for } t \geq T(t) .\end{cases}
$$

Then the boundary value problem (4.1) has at least one solution $x$ such that (2.10) and (2.11) hold.

Note that an interesting particular case is the one where the delay $T(t)$ is a nonnegative real constant.

Example Consider the second-order nonlinear delay differential equation of EmdenFowler type

$$
\left\{\begin{array}{l}
x^{\prime \prime}(t)+a(t)|x(t-r)|^{\gamma} \operatorname{sgn} x(t-r)+b(t)\left|x^{\prime}(t)\right|^{\beta} \operatorname{sgn} x^{\prime}(t)=0, \quad 0 \leq t<\infty, \\
x(t)=\xi(t), \quad-r \leq t \leq 0, \\
\lim _{t \rightarrow \infty} x^{\prime}(t)=0,
\end{array}\right.
$$

where $a(t)$ and $b(t)$ are continuous real-valued functions on $[0, \infty)$, and $\gamma, \beta$ are positive real numbers. An application of Corollary 4.1 to the boundary value problem (4.2) leads 
us to conclude that if there exists a real number $c>0$ such that

$$
\int_{0}^{r}|\xi(t-r)|^{\gamma}|a(t)| d t+c^{\gamma} \int_{r}^{\infty}(t-r)^{\gamma}|a(t)| d t+c^{\beta} \int_{0}^{\infty}|b(t)| d t \leq c,
$$

then the boundary value problem (4.2) has at least one solution $x$ satisfying (2.10) and (2.11).

For illustration purpose, suppose in (4.2) we have $r=1, \gamma=2, \beta=1$,

$$
\xi(t)=t, \quad a(t)=\frac{6}{13(t+1)^{4}} \quad \text { and } \quad b(t)=\frac{1}{6(t+1)^{2}}
$$

In this case, (4.3) is reduced to

$$
\int_{0}^{1} \frac{6(t-1)^{2}}{13(t+1)^{4}} d t+c^{2} \int_{1}^{\infty} \frac{6(t-1)^{2}}{13(t+1)^{4}} d t+c \int_{0}^{\infty} \frac{1}{6(t+1)^{2}} d t \leq c .
$$

It is not difficult to verify that this inequality is equivalent to

$$
\frac{1}{13}+\frac{1}{13} c^{2}+\frac{1}{6} c \leq c
$$

which is satisfied if and only if $0.135 \leq c \leq 7.36$. By taking $c=1$, we can conclude that the boundary value problem

$$
\left\{\begin{array}{l}
x^{\prime \prime}(t)+\frac{6}{13(t+1)^{4}}[x(t-1)]^{2} \operatorname{sgn} x(t-1)+\frac{1}{6(t+1)^{2}} x^{\prime}(t)=0, \quad 0 \leq t<\infty, \\
x(t)=t, \quad-1 \leq t \leq 0, \\
\lim _{t \rightarrow \infty} x^{\prime}(t)=0,
\end{array}\right.
$$

has at least one solution $x$ satisfying

$$
-t \leq x(t) \leq t \quad \text { and } \quad-1 \leq x^{\prime}(t) \leq 1 \quad \text { for } t \geq 0
$$

\section{Competing interests}

The authors declare that they have no competing interests.

Authors' contributions

YW carried out the most part of the paper. PJYW gave the idea and revised it. All authors read and approved the final manuscript.

Author details

${ }^{1}$ School of Mathematical Science, Guangxi Normal University, Guilin, 541004, China. ${ }^{2}$ School of Electrical and Electronic Engineering, Nanyang Technological University, 50 Nanyang Avenue, Singapore, 639798, Singapore.

\section{Acknowledgements}

Project is supported by the National Natural Science Foundation of China (11061006) and the Bagui Scholars program of Guangxi. 


\section{References}

1. Agarwal, RP, O'Regan, D: An infinite interval problem arising in circularly symmetric deformations of shallow membrane caps. Int. J. Non-Linear Mech. 39, 779-784 (2004)

2. Erbe, LH, Kong, QK: Boundary value problems for singular second-order functional differential equations. J. Comput. Appl. Math. 53, 377-388 (1994)

3. Agarwal, RP, O'Regan, D, Wong, PJY: Positive Solutions of Differential, Difference and Integral Equations. Kluwer Academic, Dordrecht (1999)

4. Agarwal, RP, O'Regan, D: Infinite Interval Problems for Differential, Difference and Integral Equations. Kluwer Academic, Dordrecht (2001)

5. Bai, $D, X u, Y$ : Existence of positive solutions for boundary value problems of second order delay differential equations. Appl. Math. Lett. 18, 621-630 (2006)

6. Agarwal, RP, Philos, CG, Tsamatos, PC: Global solutions of a singular initial value problem to second order nonlinear delay differential equations. Math. Comput. Model. 43, 854-869 (2006)

7. Bai, C, Fang, J: On positive solutions of boundary value problems for second-order functional differential equations on infinite intervals. J. Math. Anal. Appl. 282, 711-731 (2003)

8. Mavridis, KG, Philos, CG, Tsamatos, PC: Multiple positive solutions for a second order delay boundary value problem on the half-line. Ann. Pol. Math. 88, 173-191 (2006)

9. Mavridis, KG, Philos, CG, Tsamatos, PC: Existence of solutions of a boundary value problem on the half-line to second order nonlinear delay differential equations. Arch. Math. 86, 163-175 (2006)

10. Diekmann, O, van Gils, SA, Verduyn Lunel, SM, Walther, HO: Delay Equations: Functional, Complex, and Nonlinear Analysis. Springer, New York (1995)

11. Hale, JK, Verduyn Lunel, SM: Introduction to Functional Differential Equations. Springer, New York (1993)

12. Azbelev, N, Maksimov, V, Rakhmatullina, L: Introduction to the Theory of Linear Functional-Differential Equations. World Federation Publishers Company, Atlanta (1995)

13. Azbelev, N, Rakhmatullina, L: Theory of linear abstract functional-differential equations and applications. Mem. Differ. Equ. Math. Phys. 8, 1-102 (1996)

14. Schauder, J: Der Fixpunktsatz in Funktionenräumen. Stud. Math. 2, 171-180 (1930)

15. Banach, S: Sur les opérations dans les ensembles abstraits et leur application aux équations intégrales. Fundam. Math. 3, 133-181 (1922)

16. Avramescu, C: Sur l'existence des solutions convergentes de systèmes d'équations différentielles non linéaires. Ann. Mat. Pura Appl. 81, 147-168 (1969)

doi:10.1186/1687-2770-2013-14

Cite this article as: Wei and Wong: Existence and uniqueness of solutions for delay boundary value problems with p-Laplacian on infinite intervals. Boundary Value Problems 2013 2013:141.

\section{Submit your manuscript to a SpringerOpen ${ }^{\circ}$ journal and benefit from:}

- Convenient online submission

- Rigorous peer review

- Immediate publication on acceptance

- Open access: articles freely available online

- High visibility within the field

- Retaining the copyright to your article 